\title{
THE INFLUENCE OF RED AND INFRARED LIGHT ON A CRYSTAL COUNTER
}

\author{
by H. A. VAN DER VELDEN and G. P. FREEMAN \\ Physisch Laboratorium der Rijksuniversiteit te Utrecht, Nelerland
}

\section{Synopsis}

The influence of red light in the reduction of the space charge built up in a crystalcounter, is described, especially for the case of a diamond crystal. The distribution of the pulses and the decreasing of the pulse-magnitude as a function of the irradiation with $\alpha$-particles, as well as the increasing of this magnitude with red light, is explained qualitatively. It is possible to maintain the counting property of the crystal for an unlimited time, if only the intensity of the light is sufficient.

1. Introduction. An important drawback in using a crystalcounter for counting as as well for measuring energies of $\alpha, \beta$ or $\gamma$ radiation is the decrease of the number of particles or quanta, counted pro minute, as a function of the time of radiation. However not only the number of particles is decreasing but the average magnitude of the observed charge pulses is diminishing too. The lowering of the magnitude and in consequence the decrease in number can be understood if one assumes that an electric field is created, opposite in sign to the original field applied, as a consequence of the irradiation of the crystal.

To understand this, let us consider a crystal (fig. 1) with two parallel sides, thickness $d$, showing induced conductivity by $\alpha$ - or $\beta$-particles, $\gamma$-rays or light. If one ir- $\alpha$ radiates this crystal e.g. with $\alpha$-particles, at the negatively charged side, each $\alpha$-particle will send a number $(n)$ of electrons in the conduction band of the crystal and in consequence of the applied field these electrons $\alpha$ particles

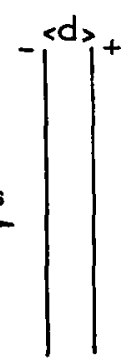

Fig. 1. travel a distance $l$ in the crystal. In that case, if one does not count a 
possible displacement of the pos. holes which are left, one finds ${ }^{1)}{ }^{2}$ ), a charge $Q=e \sum_{i=1}^{n} l_{i} / d$. Here we have added up the individual contributions of the electrons. This charge-pulse $Q$ can then be measured as a voltage change $V=Q / c$ over the crystal, if $c$ is the capacity of the crystal. Thus we see - and this also occurs with other radiations that a space charge has built up in the crystal. This would not be the case if the electrons going to the anode left the crystal (in a strong field e.g.) and if the positive charge left behind would be compensated by entering electrons. It was shown however by experiments by $\mathrm{Gudden}$ and $\mathrm{Poh}^{2}$ ) with ultraviolet light that this does not happen in general. We observed that the decrease of the number of $\alpha$ particles with time might be countered by an intense radiation of red and infrared light. The experiments described in this paper have been performed with diamonds.

Already in earlier papers methods have been considered for removing the space charge effects. Originally the methods based on irradiation with red light were used by $\mathrm{Gudden}$ and $\mathrm{Poh} \mathrm{l}^{3}$ ) during their investigation of the foto-electrical properties of various crystals in ultraviolet light. In the more recent investigations concerning the use of foto-electrical materials as counters, and also in the investigation of the conductivity changes occurring with the bombardment by electrons of low energy, other - to our opinion less simple-methods are being used. $\mathrm{V}$ a $\mathrm{n} \mathrm{H}$ e e $\mathrm{rde}^{1}$ ) irradiated his $\mathrm{AgCl}$ crystal after use with red light. Wouters and $\mathrm{Chr}$ is$t \mathrm{i} a \mathrm{n}^{4}$ ) indicate that it is possible to reverse the voltage on the $\mathrm{AgCl}$ crystal after a certain number of counts, when detecting highenergy particles, and $\mathrm{Mc} \mathrm{K} \mathrm{a}{ }^{5}$ ), in his investigation of the conductivity change of diamonds during the irradiation with electrons of low energy, changes the circumstances in a rather complicated way and assumes in his case that for the maximum value of $Q$ the optimum decrease of the space charge is reached. $\mathrm{M} \mathrm{c} \mathrm{K}$ a y measures the effect of pulses, consisting of a number of mono-energetical electrons. The space charge is diminished by using an alternative voltage on the crystal; the electronpulses are given at the moment of each (pos. or neg.) maximum of the voltage. But furthermore these electronpulses are superimposed on a constant current of irradiation electrons. Of course the effect of this current will not be measured by the amplifier, but only the current strength is chosen such as to make the deflection (the $Q$ ) of each pulse an optimum. 
2. Experimental arrangement. To measure the charge pulses given by separate particles we used a diamond on which aluminium electrodes had been deposited by evaporation. The diamond was mounted in a special holder. One of the electrodes was connected to a high voltage apparatus. This voltage was measured with an electroscope, the other electrode was connected with the grid of a first valve of an ionisation-chamber amplifier. With the aid of a grid-battery, it was possible to maintain the potential of this grid on a average constant value. The crystal holder was constructed in such a way that charge pulses on the grid, caused by the ionisation of the air at the sides of the diamond were prevented (fig. 2). The lineair ionisation-

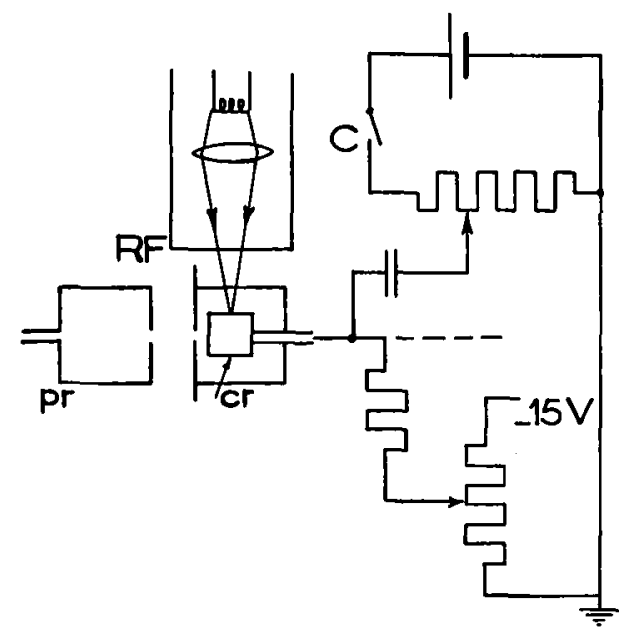

Fig. 2. The diamond crystal counter and the calibrationcircuit. $p r=$ preparation of $\alpha$-particles; $c r=$ crystal; $R F=$ red filter; $c=$ calibration circuit.

chamber amplifier was constructed for minimum noise. Full particulars of it have already been published by $\mathrm{v}$ a $\mathrm{n} \mathrm{He}$ e $\mathrm{rde} \mathrm{n}^{\mathbf{1}}$ ) and $\left.\mathrm{Kelle}{ }^{6}\right)$. The output of the amplifier was connected to the vertical deflection plates of an oscillograph and at the same time via a gastriode to a multivibrator, whose output acted as a time base for each pulse, and the total deflection-time of this time-base was of the same duration as the time of the signal so as to give us the possibility of photographic registration of the deflection.

The maximum deflection (see $\mathrm{van} \mathrm{He}$ e $\mathrm{de} \mathrm{n}$ ) is proportional to the charge pulse $Q$. The dual function of the gastriode is to give 
signals of constant magnitude to the multivibrator, and to suppress the noise of the amplifier by an negative voltage on its grid. To measure the absolute value of $Q$ we used a calibration circuit consisting of a $1 \mathrm{~cm}$-condensor, a variable resistance, an accu and a mercury-switch, in the same way as $\mathrm{v}$ a $\mathrm{n} \mathrm{H}$ e e r de $\mathrm{n}$ did.

Contrary to the method of the much used discriminator and scales, we registered our results photographically. The reasons were, primary: we could verify our results afterwards; secondly: in our case it was cheaper and when compared with many channel discriminations with counting circuits, simpler and more reliable; and last: we could eliminate such parts which, influenced by external disturbances, would give a mutilated picture of the measurements.

Between the multivibrator and the horizontal deflection plates we used one or two scales, depending on the intensity of the $\alpha$-radiation, for the purpose of recording only 1 out of $8,16,32$, or 64 deflections of the entering $\alpha$ particles. This was necessary in order to maintain a reasonable speed of the recording drum, even at an intense radiation; this intense radiation was important to obtain a decrease in the number of the counts within a reasonable time.

Both the zeropoint of the oscilloscope and the noise were suppressed. In order to prevent burning of the oscilloscope and blackening of the recording paper the zeropoint was suppressed by applying a negative voltage to the $\mathrm{W}$ e h n e l t cylinder of the oscilloscope valve; this negative voltage was elevated during the time of the signal by a positive square voltage pulse, given by the multivibrator. The noise was suppressed, as we told already, by a negative voltage on the grid of the gastriode.

The red light was produced by a projection lamp ( $60 \mathrm{Watt}$ ). The light was concentrated on one side of the diamond by means of a strong lens and via a red filter. At the onset of our experiments we used a filter, which cut off sharply the light below $6000 \AA$, later on we used another filter, cutting down at $6900 \AA$.

3. The measurements. The decrease of the number of counts during the proceedings of the irradiation without the red light is illustrated in fig. 3. Usually in the following experiments we used a radiation of nearly 3000 particles per minute on a surface of $6 \mathrm{~mm}^{2}$, while the efficiency of the diamond used in the experiments mentioned below, was about $25 \%$. Quantitatively however, the decrease 
and subsequent increase to the original level after irradiation with red light was observed with more diamonds. The decrease magnitude was nearly unobservable during the time that the number was a constant. Under these circumstances, while the voltage on the crystal was varied between 350 and $1000 \mathrm{~V}$, the time of irradiation $\left(T \frac{1}{2}\right)$ necessary to reduce the number pro minute to one half, ranged between half an hour and two hours and was roughly linear with the voltage. But if one irradiated afterwards with red light, the number $\mathrm{N} / \mathrm{min}$. within a short time (a few minutes) increased to the original level. At first we used a rather weak lightsource $(20 \mathrm{Watt})$ and poor optics; then the number also increased, but later decreased again in the way sketched in fig. 4 . Here the values of the horizontal part are the same as those before the irradiation.

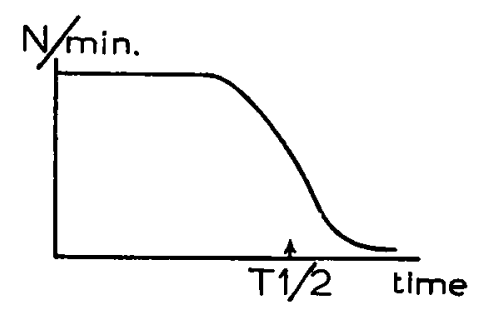

Fig. 3. The decrease of the number of counts (in absence of red light).

Then we passed on to a more intense irradiation with red light, namely to a $60 \mathrm{Watt}$ projection lamp and better optics. The same filter (absorbing all light below $6000 \AA$ ) was used. Then we observed no decrease for several voltages up to a time of seven hours and after switching off the red light it lasted a considerably longer time than in the preceding experiments before the decrease became apparent again. Furthermore we observed that in general the distribution of the height of the signals in a long-time irradiation changed in such a sense that for a constant voltage we obtained a relatively smaller number of smaller signals than at the beginning of the experiments.

The limit of about seven hours to which the experiments were bound, was caused by a rather sudden decrease of the potential of the floating grid of the first valve of the amplifier. Evidently we still got a detectable foto-electrical current for wavelengths above the $6000 \AA$ wave-length of the red light; for this reason we afterwards used a higher wave-length $(6900 \AA)$ and then this effect ceased. The 
result of our observations is obviously that with the aid of red light a diamond (or other crystal) can be used for un unlimited time in the counting of $\alpha$-particles (or particles of high energy). An attempt to get a same effect with $\beta$-particles was not continued, owing to the weak intensity of the only $\beta$-source at our disposal at that time. This intensity was insufficient to obtain a detectable decrease within a reasonable time.

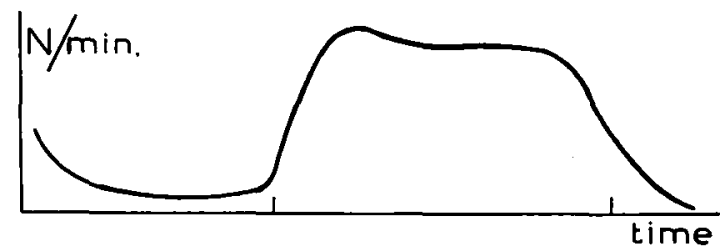

Fig. 4. The number of counts per minute, while the irradiation with red light is insufficient.

4. Discussion. We should like to remark, that for an explication of the sudden decrease in the quantity $Q=e \sum_{1}^{n} i l_{i} / d$ already mentioned, we think especially of a diminuation of $l_{i}$, the distance covered in the crystal. This could be explained by the formation of a strong field, opposite to the initial one. In accepting this, there is a difficulty - which we hope to solve in a next paper - because in the first place we ought to expect a slow decrease in the magnitude of the signal. As the experiments of $\mathrm{Gudden}$ and $\mathrm{Pohl}{ }^{2}$ ) and of $\mathrm{M} \mathrm{c} \mathrm{K} \mathrm{a} \mathrm{y}{ }^{5}$ ) show, the saturation field strength for diamonds (that is the field strength causing all or nearly all the electrons to travel through the whole of the crystal) is higher than the values, used in our experiments. However the decrease in itself, even if it is a bit faster than according to these older publications, would not explain the decrease of the number in such a short time. This would be more understandable if one assumes that in general the diamond, instead of being a perfect monocrystal, is a polycrystal built up of little crystals, which when irradiated fall out one after another and then indeed by the forming of an opposite field and on the average at the same time.

This explanation is in accordance with earlier experiments which show that ultraviolet-transparent crystals posses a laminar structure ${ }^{7}$ ). The crystal we used was, in effect, selected by the ultraviolet- 
transparence test. Another explanation, saving the monocrystalstructure for diamond, as far as we understand is not available.

In addition we should like to discuss the reason why the red and infrared light is able to maintain a reasonable electric field in the crystal. Lightquanta are able to liberate electrons from traps to such an extent, that they will travel in a local field, be catched again, and partly compensate positive holes. The fraction liberated in this way and the part which reaches the anode, together are evidently big enough, if the crystal is exposed to sufficient irradiation with red light, to create a resulting field in the crystal in which the electrons travel over a distance comparable with that in the initial external field.

In this picture, however, without a more detailed explication it is not clear why with insufficient irradiation by red light, after the blocking of the crystal in absence of red light the number of counts should increase rapidly to the original number and after some time should decrease again. Why can the red light make an original change in the field to such an extent (and so fast), while some time later it cannot do this any more?

A possible explanation of this fact may be that there are traps of different depths. One can expect that after the space charge has been built up in absence of irradiation, part of the filled traps are shallow enough to have their electrons liberated immediately after irradiation with red light. From this moment onwards these traps are not effective any more in producing space charge and a new space charge is built up by electrons captured in deep traps which have only a small chance to be released by red light. So a new situation results in which in insufficiently strong red light the space charge might reduce the number of pulses again after some time. In a sufficient amount of red light however an equilibrium is reached in which the number of new electrons trapped equals the number released. This equilibrium depends on the number of $\alpha$-particles pro minute and on the intensity and composition of the light.

5. Conclusion and comparison with literature. The experiments, described in this paper, prove the possibility to obtain a fargoing reduction of the space charge effect in a crystal counter. The method is simpler than the one used by $\mathrm{M} \mathrm{c} \mathrm{K}$ a y in his investigation of the conduction of diamond caused by irradiation with low-energy elec- 
trons. Moreover, by the use of $\alpha$-particles, it offers the opportunity to determine a number of characteristics of crystalcounters, which is an important possibility both for the theory of the foto-electrical phenomena and for the use of the crystalcounter.

In future papers we hope to publish the results of experiments with other diamonds and in other circumstances, as well as the extension of the experiments described above.

To explain our experiments we needed three assumptions; primary: that diamond is not a monocrystal; secondly: the results of $\mathrm{Gudden}$ and $\mathrm{Pohl}$ and $\mathrm{McKay}$ on the fieldstrength-dependance of the fotocurrent are not quite correct; and lastly: the irradiation of the red light affects the space charge caused by the electrons in shallow traps. The first idea is confirmed by experiences in the X-ray examination of diamond. After the conclusion of these experiments, we received a copy of the Physical Review, in which $\mathrm{Chynowe} \mathrm{h}^{8}$ ) in a letter to the Editor points out the importance of the irradiation with infrared light to the use of a crystalcounter. His experiment was also carried out with $\alpha$-particles and a diamond and his result appears to agree with our experiences as to the constant number of the counted particles during the irradiation and the decrease after the switching off of the infrared radiation. As fas as we understand the extension of his experiment will be more in the direction of an investigation of the depths of traps, to be carried out by varying the wave lenght of light.

Our thanks are due to Professor M i l a t z for his highly estimated and stimulating interest during the course of this investigation, and to the "Stichting Fundamenteel Onderzoek der Materie" that made these experiments possible under assistance from the ,Nederlandse Organisatie voor Zuiver Wetenschappelijk Onderzoek".

Received 19.5.50.

\section{REFERENCES}

1) P. J. va n He e r d e n, The crystal counter, Thesis, Utrecht 1945.

2) B. Gudde $n$ und R. Po h l, Z. Phys. 16, 170, 1923.

3) B. Gudden und R. Poh 1, Z. Phys. 17, 331, 1923.

4) L. F. Wouters and R.S. Christia n, Phys. Rev. 72, 1127, 1947.

5) K. G. M c K a y, Phys. Rev. 7A, 1606, 1948.

6) K. J. Ke 11 e r, Thesis, Utrecht 1945.

7) C. V. R a m a n et al., Proc. Ind. Acad. Sci. Symposium I, 19A, 1944;Symposium II, 24A, 1946; see too: R. H of s t a d t e r, Phys. Rev. 73, 631, 1948.

8) A. G. Chy now e th, Phys. Rev. 76, 310, 1949. 